Laetitia L.C. Prestoz Klaus G. Petry

\section{ADRESSES}

L.L.C. Prestoz: Wellcome/CRC Institute, Tennis Court road, Cambridge CB2 1QR, Royaume-Uni. K.G. Petry: Laboratoire "neurobiologie des affections de la myéline " EA 2966, Université Victor-Segalen Bordeaux 2, 146, rue Léo-Saignat, 33076 Bordeaux, France.

\title{
Un siècle d'étude de la galactosémie
}

Le déficit en galactose-1-phosphate uridyl transférase (GALT), responsable de la galactosémie "classique", est dû aux mutations du gène codant pour cette enzyme. Chez le nouveau-né, alors qu'un régime alimentaire sans galactose élimine la symptomatologie aiguë, les affections chroniques - retard intellectuel et troubles de la reproduction persistent. Des études récentes ont permis d'associer la galactosémie au syndrome de déficience en hydrates de carbone des glycoprotéines. L'ensemble des recherches actuelles concernant la pathogénie de la galactosémie vise essentiellement (1) à mettre en place des stratégies thérapeutiques afin d'éviter l'apparition des troubles neurologiques et endocriniens par une limitation de galactose et de ses dérivés dans l'organisme et (2) à rechercher un traitement qui pourrait stimuler des voies secondaires du métabolisme afin de métaboliser les dérivés toxiques du galactose et d'augmenter le taux de l'UDP-galactose, nécessaire à la galactosylation des protéines et des lipides.

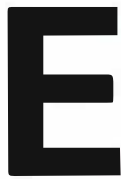
raison de la structure très hétérogène et des rôles diversifiés des glycanes, l'altération de la structure de ces composés est à l'origine d'un grand nombre de maladies. I a plupart de ces affections est liée à une perturbation du catabolisme des oligosaccharides dont les plus connues sont les mucopolysaccharidoses, les glycoprotéinoses, ou encore les glycolipidoses. Plus récemment, des maladies affectant la synthèse des chaînes glycaniques des glycoprotéines ont été mises en évidence. Une grande partie de ces maladies, caractérisées par une déficience en hydrates de carbonc des glycoprotéines du plasma, a été regroupée sous le nom de syndromes de déficience des glycoprotéines en hydrates de carbone ou syndromes CDG [1].

I.es syndromes CDG sont caractérisés par des manifestations cliniques multisystémiques plus particulièrement accompagnées de perturbations du système nerveux central et périphérique. Ils ont un caractère héréditaire et se transmettent sur un mode autosomique récessif. L'ensemble de ces maladies présente de nombreuses caractéristiques génétiques, biochimiques et cliniques communes à celles observées chez. des patients atteints de galactosémie classique [2]. En effet, plusieurs travaux mon- 
trent que la galactosémie est également associée à une altération de la synthèse des chaines glycaniques de certains glycolipides et de glycoprotéines [3-7].

La galactosémie a été décrite pour la première fois en 1908 par von Reuss [8]. Une dizaine d'années plus tard, cette maladie est reconnue comme un dysfonctionnement du métabolisme du galactose [9], mais il faudra cependant attendre près de 40 ans pour que l'origine de cette maladie soit attribuée à une déficience génétique de l'enzyme galactose-1-phosphate uridyl transférase (GALT) [10, 11]. Cette affection est transmissible sur le mode autosomique récessif. Elle est caractérisée par la présence de mutations ponctuelles et de polymorphismes sur le gène codant pour l'enzyme GALT [12]*, se traduisant soit par une perte totale d'activité (galactosémie classique), soit par une activité résiduelle plus ou moins importante: de $1 \%$ à $15 \%$ de la normale chez les variants symptomatiques (galactosémie modérée), pouvant atteindre $50 \%$ à $150 \%$ de la normale che $\%$ les variants asymptomatiques [13].

\section{L'enzyme GALT a une position centrale au sein du métabolisme du galactose}

Cette enzyme catalyse la deuxième étape de transformation du galactose en glucose, en assurant la production d'UDP-galactose et de glucose-1phosphate à partir d'UDP-glucose et de galactose-1-phosphate (figure 1). Ce sucre nucléotidique est essentiel puisqu'il est le seul donneur de résidus galactosyls nécessaires à la synthèse des chaines d'hydrates de carbone des glycoprotéines et des glycolipides [13]. Ainsi, une déficience enzymatique de GALT entraîne un déséquilibre du métabolisme du galactose caractérisé par une accumulation de galactose-1phosphate et d'autres composés dérivés tels que le galactitol ou le galactonate et une altération de certains glycoconjugués galactosylés [3-7]. Les réactions enzymatiques du métabolisme du galactose font également

* Voir la liste des mulations sur le site 786

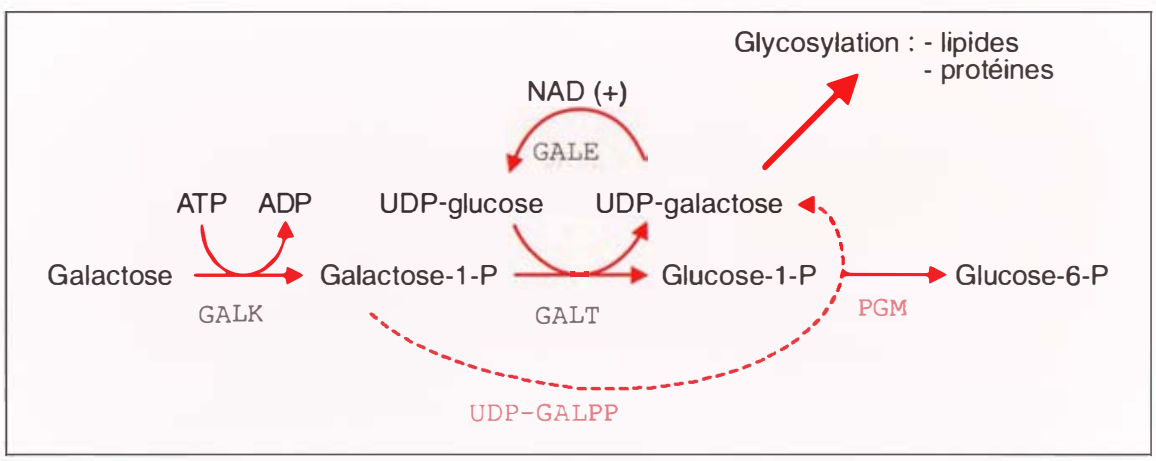

Figure 1. Représentation schématique des voies du métabolisme du galactose. Le galactose est métabolisé par trois enzymes principales: galactokinase (GALK), galactose-1-phosphate uridyl transférase (GALT) et UDP-galactose-4'-épimérase (GALE). Une déficience de chacune de ces enzymes donne lieu à un type particulier de galactosémie. Le type de galactosémie présentant les symptômes les plus graves est associé à une déficience enzymatique en GALT. Une voie métabolique secondaire catalysée par l'enzyme UDPgalactose pyrophosphorylase (UDP-GALPP) assure la transformation du galactose-1-phosphate en UDP-galactose, utilisé pour la glycosylation de lipides et de protéines. La voie catalysée par l'enzyme phosphoglucomutase (PGM) permet la transformation du glucose-1-phosphate en glucose-6-phosphate, ensuite métabolisé au cours de la glycolyse.

intervenir deux autres enzymes ubiquitaires intracytoplasmiques (galactokinase ou GALK et UDP-galactose4'-épimérase ou GALE) (figure 1) dont la déficience respective se rapporte à un type particulier de galactosémie. La déficience en GALT ne représente pas la forme la plus répandue de la galactosémie (1 nouveau-né sur 40000 à 60000 est atteint selon sa population d'origine) [13] mais ce type de galactosémie dite "classique" présente les manifestations cliniques à long terme les plus importantes, à savoir de graves troubles neurologiques et endocriniens.

\section{Variabilité et sévérité des symptômes cliniques}

Les manifestations cliniques précoces d'une déficience totale en GALT apparaissent dès l'ingestion de lait dans les premiers jours de vie. Elles se traduisent par des symptômes caractéristiques d'une défaillance hépato-rénale avec notamment des vomissements fréquents, un ictère et une hypoglycémie [14]. De plus, des taux très élevés de galactose-1-phosphate ainsi que de galactitol peuvent être détectés dans les tissus, le sang et les urines. Une septicémie à Escherichia coli est parfois observée. Ces troubles peuvent conduire au décès de l'enfant. En l'absence de toute intervention thérapeutique, l'enfant survivant développe un hépatopathie chronique (cirrhose), un retard mental et une cataracte bilatérale [13]. Afin d'éviter à ces nouveau-nés une issue fatale, un régime alimentaire sans galactose a été proposé [15]. A l'époque, ce traitement a suscité beaucoup d'espoirs, puisque l'élimination presque totale du galactose dans l'alimentation a permis de spectaculaires régressions de la maladie. Pendant les premières années de vie, les bénéfices de ce régime ont paru évidents: les manifestations néonatales disparaissent et ce régime alimentaire assure un développement staturo-pondéral normal des nouveau-nés.

Les premiers doutes sur l'efficacité du traitement sont apparus en 1969 [16]. En effet, malgré ce régime, les taux de galactose-1-phosphate restaient anormalement élevés dans le sang et les urines des patients [17]. La désillusion s'est confirmée au cours des années 1980, quand des déficits cognitifs et des troubles de l'appareil reproducteur ont été mis en évidence chez les enfants traités [18]. Il apparaît maintenant que le régime alimentaire sans galactose n'est pas suffisant pour assurer un 


\begin{tabular}{|l|l|}
\hline \multicolumn{1}{|c|}{$\begin{array}{c}\text { Tableau I } \\
\text { COMPLICATIONS À LONG TERME MISES EN ÉVIDENCE } \\
\text { CHEZ LES PATIENTS ATTEINTS DE GALACTOSÉMIE CLASSIQUE }\end{array}$} \\
\hline \multicolumn{1}{|c|}{ Déficience enzymatique en GALT (régime alimentaire sans galactose) } \\
\hline $\begin{array}{l}\text { Troubles neurologiques } \\
\text { - diminution du QI avec l'âge } \\
\text { - dyspraxie verbale } \\
\text { - retard de croissance } \\
\text { - microcéphalies } \\
\text { - ataxies } \\
\text { - dégénérescence neuronale corticale } \\
\text { - atrophie du cervelet et des ganglions de la base } \\
\text { - épilepsies }\end{array}$ & Troubles endocriniens \\
\hline
\end{tabular}

développement normal des patients, ceux-ci présentant encorc des troubles neurologiques caractéristiques d'une maladie neurodégénérative et des troubles endocriniens affectant le système reproducteur (Tableau I).

\section{Troubles neurologiques}

De nombreuses études longitudinales ont été menées chez un grand nombre de patients. Toutes convergent vers les mêmes observations mettant en évidence un développement mental clairement compromis même chez les patients suivant rigoureusement le régime imposé [14]. Le quotient intellectuel diminue progressivement avec l'âge, démontrant une perturbation lente et progressive des fonctions cérébrales. Plus de la moitié des patients présentent des problèmes de dyspraxie verbale et un retard de croissance important [14, 19]. Des signes caractéristiques de maladic neurodégénérative tels qu'une microcéphalie, des tremblements et une ataxie ont également été observés. Les fonctions motrices sont perturbées, les patients ont des difficultés à se mouvoir et à se maintenir en équilibre [20]. Des coupes histologiques de cerveau et des études en imagerie par résonance magnétique montrent de surcroît une dégénérescence neuronale corticale, une atrophie du cervelet et des ganglions de la base ainsi qu'une altération de la myéline [21, 22]. Enfin, quelques cas d'épilepsie ont été rapportés [23]. Aucune corrélation n'a pu être mise en évidence $\mathrm{m} / \mathrm{s} n^{\circ}$ 6-7, vol. 16, juin-juillet 2010( entre l'apparition de ces symptômes, l'âge auquel le patient a débuté le régime alimentaire sans galactose et la présence ou non de symptômes néonatals [20]. De même, les taux de galactitol et de galactose-1-phosphate ne sont pas corrélés au quotient intellectuel mesuré.

Dans le cas d'une galactosémie classique modérée, c'est-à-dire chez des patients possédant une activité résiduelle de GALT de $1 \%$ à $15 \%$ de la normale, aucune anomalie de croissance ni de développement mental n’a été observée [22].

\section{Troubles endocriniens}

Les troubles endocriniens sont essentiellement caractérisés par des troubles de la fonction reproductrice. En effet, chez $66 \%$ [24] à $92 \%$ [25] des patientes ne présentant aucune activité GALT, un dysfonctionnement ovarien caractérisé par un hypogonadisme hypergonadotrophique a été observé $[14,20]$. Ce dysfonctionnement s'accompagne de concentrations anormalement élevées d'hormones gonadotropes (l'hormone folliculo-stimulante [FSH] et l'hormone lutéinisante $[\mathrm{LH}]$ ) et anormalement faibles d'œstradiol dans le sérum des patientes et cela dès l'âge de un an [24].

Les patientes présentent plus particulièrement une atrophie des follicules ovariens due à une accumulation précoce des métabolites du galactose dans le parenchyme ovarien [26] ainsi qu'une altération structurale des isoformes de $\mathrm{FSH}$ plasmatiques [7]. Ces isof ormes de FSH, précisé- ment déficientes en galactose et par conséquent en acide sialique (saccharide terminal des chaînes d'hydrates de carbone de la $\mathrm{FSH}$ ) pourraient être en partie responsables de l'hypogonadisme observé. En effet, de telles isof ormes sont incapables d'entraîner une réponse intracellulaire en se fixant sur leurs récepteurs ovariens [27]. L'atrophie des ovaires associée à la présence d'isoformes de FSH altérées peuvent ainsi expliquer les faibles taux d'œstrogènes observés dans le plasma des patientes. Cependant, les mécanismes qui sous-tendent ce dysfonctionnement restent à élucider.

En outre, une importante variabilité individuelle caractérise ces troubles. En effet, ceux-ci peuvent se traduire par des aménorrhées primaires ou secondaires et une stérilité partielle ou totale des patientes. Il semblerait qu'une activité résiduelle de GALT minime soit suffisante pour assurer le bon fonctionnement de l'appareil reproducteur, comme l'illustre le cas d'une patiente ayant une activité résiduelle inférieure à $2 \%$ de la normale [25]. De plus, sans que l'on en connaisse encore l'explication, l'analyse de certains cas a montré que le dysfonctionnement ovarien pouvait être réversible chez quelques patientes sous régime alimentaire sans galactose [28]. Au contraire la fécondité épargnée des hommes atteints de galactosémie classique est une chose surprenante. En effet, seulement 3\% de ces patients présentent des taux anormaux d'hormones gonadotropes. Des travaux récents montrant que des animaux mâles déficients en FSH sont 
féconds malgré un faible développement de leurs organes génitaux [29] pourraient expliquer l'absence de troubles de l'appareil reproducteur che\% les hommes atteints de galactosémie. Enfin, l'hypogonadisme hypergonadotrophique peut apparaître très précocement chez les femmes malades puisqu'il a ćté observé dès l'âge de neuf mois chez une patiente attcinte de galactosémie n'ayant jamais été exposée au galactose exogène lors de sa vie in utero et après la naissance [30].

\section{Pathogénie de la galactosémie: les différentes hypothèses}

L'étiologie des complications à long terme caractéristiques de la galactosćmie, constitue unc véritable énigme depuis près de trente ans. De nombreuses études de cas galactosémiques tentent d'élucider la pathogénic de cette maladic et ainsi de trouver les mécanismes associés aux complications neurologiques et endocriniennes observées.

Deux principales hypothèses, non exclusives l'une de l'autre, permettent d'expliquer les mécanismes d'apparition des troubles neurologiques et endocriniens $[13,31]$. Si ces deux hypothèses s'accordent sur la nature du déficit enzymatique en GALT, l'une met en relief l'excédent endogène et/ou exogène du substrat enzymatique (le galactose et ses dérivés métaboliques) alors que l'autre incrimine le déficit du produit de la réaction enzymatique mise en cause (notamment l'UDP-galactose) (figure 2).

\section{L'intoxication par le galactose et ses dérivés métaboliques}

L'intoxication par le galactose et ses dérivés métaboliques peut être soit de type dit "endogène", ou de type " exogène". Ia thèse de l'intoxication "endogène " ou «auto-intoxication" a été développée par Gitzelmann et al. en 1975 [32] et récemment renforcée [33]. En effet cette thèse a été proposée après avoir observé que des patients, malgré un régime strict sans galactose, présentaient dans leurs urines des taux élevés de galactitol et de galactonate nique de galactose-1-phosphate dans leurs globules rouges. Le galactose pourrait provenir soit de la digestion ou du clivage de l'UDP-galactose par l'enzyme UDP-galactose pyrophosphorylase (figure I), soit du renouvellement ou du catabolisme des hydrates de carbone galacto-lipidiques et galacto-protéiniques. Les molécules de galactose ainsi produites seraient ensuite métabolisées grâce à des voies secondaires du métabolisme transformant le galactose en dérivés galactonate et galactitol, ce dernier composé s'avérant toxique pour l'organisme à fortes doses [34].

Une autre hypothèse permettant d'expliquer l'accumulation des dérivés du galactose chez les patients atteints de galactosémie classique et suivant un régime sans galactose, est l'intoxication par le galactose exogène. En effet, des sources cachées de galactose libre dans certains éléments de notre alimentation comme les fruits et les légumes ont été mises en évidence [35], rendant ainsi difficile la mise en place d'un régime alimentaire totalement dépourvu de galactose.

Outre la toxicité due à l'accumulation de dérivés toxiques, la présence d'un excès de galactose pourrait également entraîner un déficit métabolique. En effet, l'administration à des rats de fortes doses de galactose induit une diminution des taux d'inositol dans le cristallin et le nerf sciatique de ces animaux [36]. L'excès de galactose pourrait ainsi provoquer une perturbation du métabolisme des phosphatidyl-inositols et par conséquent une altération de la transduction de signaux intracellulaires.

Enfin, certains travaux ont montré que le galactose-1-phosphate peut inhiber l'activité endogène d'enzymes galactosyltransférases lorsqu'il est à forte concentration dans la cellule [37]. Comme ce type d'enzyme

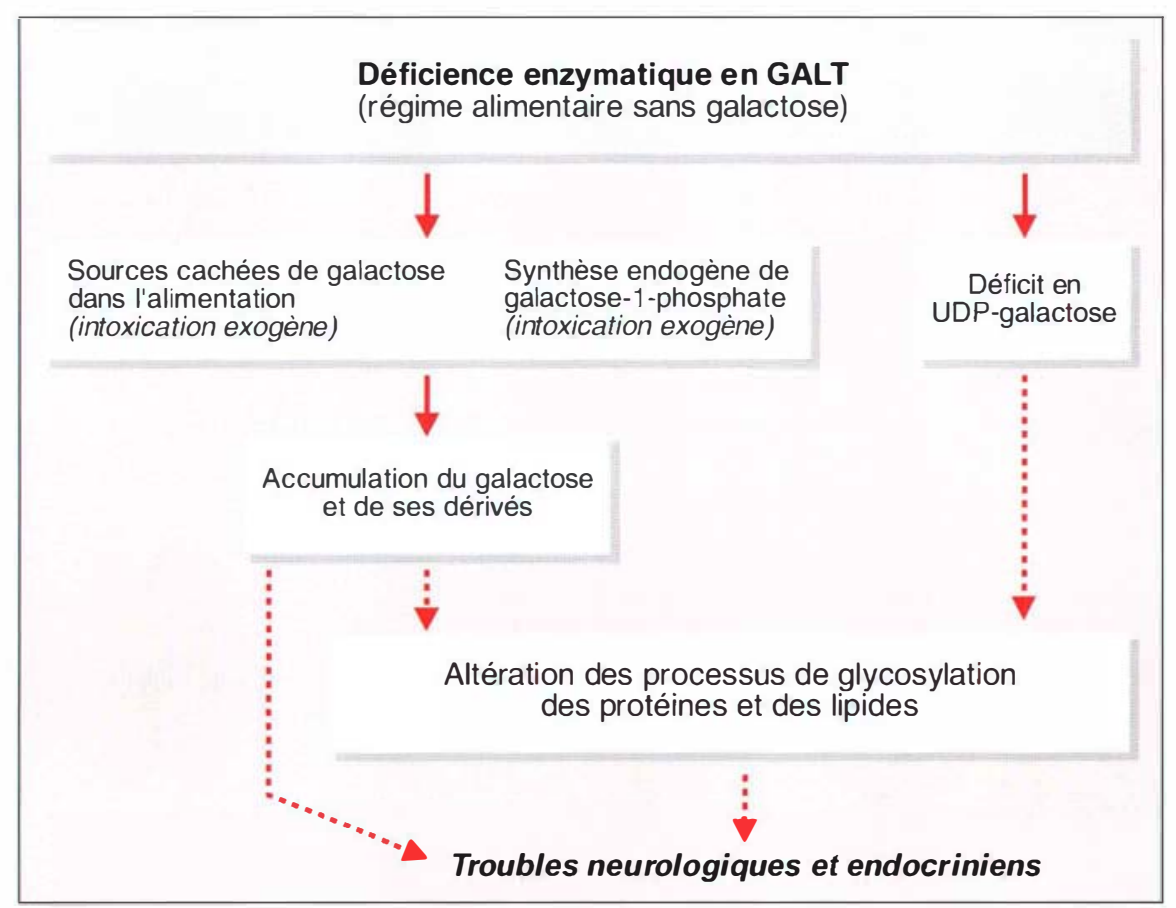

Figure 2. Mécanismes pathogéniques de la galactosémie. Malgré un régime alimentaire sans galactose, une déficience enzymatique en GALT est caractérisée par une accumulation de galactose et de ses dérivés métaboliques (provenant de sources endogènes et/ou exogènes) et un déficit en UDP-galactose. Les recherches actuelles montrent que ces deux phénomènes pourraient induire une altération des processus de glycosylation, entraînant ainsi les troubles neurologiques et endocriniens observés. De plus, l'accumulation de galactose et de ses dérivés métaboliques a un effet toxique sur l'organisme et pourrait ainsi participer directement à l'apparition des troubles. 
assure le transfert de résidus galactose sur les chaînes d'hydrates de carbone des glycoprotéines et des glycolipides, une accumulation de galactose-1phosphate peut entraincr une perturbation de la galactosylation de ces structures. Cette hypothèse permettrait d'expliquer ainsi l'hypoglycosylation observée pour certaines molécules dans les fibroblastes en culture de patients atteints de galactosémie $[3,4,6,23]$ et une déficience en glycolipides contenant du galactose ou clu N-acétylgalactosamine [5] ainsi qu'une hypogalactosylation des molécules de FSH [7]. Une hypoglycosylation des chaînes d'hydrates de carbone de la transferrine plasmatique a également été mise en évidence che\%. des patients atteints de galactosémie, cette perturbation se normalisant presque totalement au cours de l'instauration d'un régime dépourvu de galactose [38]. Enfin, nos récents travaux ont mis en évidence une altération de la glycosylation de molécules de FSH clans le plasma de rates soumises à un régime enrichi à $40 \%$ en galactose. L'ensemble de ces données s'accordent donc sur l'influence d'une intoxication par le galactose sur les processus de glycosylation de glycoconjugués.

\section{Déficit métabolique en UDP-galactose}

Le phénomène d'intoxication par le galactose endogène ou exogène n'exclut pas la possibilité de déficits métaboliques. Cette diminution du taux d'UDP-galactose a été mesuré dans les érythrocytes et les leucocytes de patients atteints de galactosémie classique [3]. Compte tenu du fait que l'UDP-galactose est le seul donneur de résidus galactosyls pour la synthèse des chaînes glycaniques [13], ce groupe de chercheurs a postulé que le déficit en UDP-galactose dû à une déficience totale de l'enzyme GALT, pouvait être responsable de l'altération des processus de glycosylation observée dans certains glycoprotéines et glycolipides. Cette hypothèse suppose que l'enryme GAL.E est incapable de compenser la déficience en UDP-galactose à partir de l'UDP-glucose (figure 1) puisque dans des conditions normales, cette enzyme permet de maintenir un rapport UDP-galactose/UDP-glucose de 1/3 dans la cellule.

$\mathrm{m} / \mathrm{s} n^{\circ} 6-7$, vol. 16, juin-juillet 20(1)(
Bien que la méthode de mesure de l'UDP-galactose ait été largement controversée et que d'importantes variabilités interindividuelles aient ćté obscrvées che\% les patients attcints de galactosémie, l'hypothèse d'un déficit en UDP-galactose n'est à l'heure actuclle pas abandonnée puisqu'aucune méthode directe de mesure de I'UDP-galactose n'a été mise en place et puisqu'il est clair que le rapport des taux de UDP-glucose et d'UDP-galactose sont anormaux chez ces patients, témoignant ainsi d'une perturbation du métabolisme de ces sucres.

\section{Orientations thérapeutiques et impératifs de recherche}

L.es recherches actuelles concernant la pathogénie de la galactosémie visent essentiellement à mettre en place des stratégies thérapeutiques afin d'éviter l'apparition des troubles neurologiques et endocriniens.

Le seul traitement réellement mis en place était fondé sur une administration d'uridine. Cette idée d'une thérapie à l'uridine est apparue dès 1989, lorsqu'il a été montré que le taux d'UDP-galactose était déficient dans les érythrocytes des patients [3]. Des études ont montré que l'administration orale d'uridine à ces patients pouvait restaurer le taux d'UDP-galactose [26]. En effet, I'uridine par le biais d'une production d'UDP-glucose, permet la synthèse d'UDP-galactose par l'enzyme GALE. Les résultats d'une étude à plus long terme sur des patients n'ont malheureusement montré aucun changement de l'atteinte des fonctions cognitives che\% les patients traités à l'uridine [39]. De plus, il s'est avéré que l'uridine pouvait avoir une action inhibitrice sur l'activité de l'enzyme GALT [40].

A l'heure actuelle, l'échec de ce traitement rend impératif la mise en place d'une alternative thérapeutique af in d'éviter l'apparition des complications neurologiques et endocriniennes. Cette alternative pourrait être envisagéc en essayant de pallier à la déficience métabolique, et/ou en tentant de limiter l'accumulation des composés toxiques.
Pallier le déficit en UDP-galactose : régulation dirigée de l'activité de l'enzyme GALE

Puisque le faible taux d'UDP-galactose semble être impliqué dans la pathogénic des troubles observés che\% les patients atteints de galactosémie classique, l'augmentation de la production de ce sucre nucléotidique apparaît immédiatement comme une solution pour pallier cette déficience.

Nos récents travaux ont mis en évidence une expression variable de l'enzyme (iALE dans la glande mammaire au cours de différents stades de la lactation che\% la rate, montrant ainsi une forte expression de cette enzyme en début et en milieu de lactation par rapport aux stades de fin de lactation et de repos de la glande mammaire [41]. Cet organe dans ces conditions physiologiques particulières peut ainsi être utilisé pour étudier les éléments régulateurs de l'expression de l'enzyme GALE. Le but serait alors de mettre en place une régulation dirigée de l'expression et de l'activation de l'enzyme afin de compenser le déficit en UDPgalactose, par une administration de produits pharmacologiques activateurs de l'enzyme.

L'enryme GALE est une enzyme bifonctionnelle qui assure notamment l'épimérisation de l'UDP-glucose en UDP-galactose. Le rapport du taux d'UDP-galactose sur le taux d'UDP-glucose est maintenu à $1: 3$ dans la cellule dans des conditions normales. Ainsi, bien que la réaction soit réversible, elle favorise cependant la production d'UDP-glucose, plutôt que celle d'UDP-galactose. Il serait donc possible d'augmenter la production d'UDP-galactose sous condition que le taux d'UDP-glucose intracellulaire soit élevé. Ainsi, l'administration de produits activateurs de l'enzyme devrait être associéc à une administration d'uridine, par exemple, ayant été montré augmenter le taux d'UDPglucose che\% les patients [26]. Les quantités intracellulaires des co-facteurs NAD et NADH nécessaires à l'activité de l'enzyme sont également des facteurs importants de la régulation de cette enzyme.

Une investigation complète des conditions de régulation de l'expression de l'enzyme reste à faire. 


\section{Limiter la présence de galactose et de ses dérivés dans l'organisme}

La seule possibilité pour diminuer l'apport de galactose exogène est d'affiner le régime alimentaire en éliminant le maximum de composants contenant du galactose.

En revanche, plusieurs possibilités pourraient être étudiées afin de limiter la synthèse endogène de galactose et l'accumulation du galactitol et du galactose-l-phosphate, que l'on soupçonne d'être responsables de la plupart des symptômes de la galactosémie.

Un traitement pourrait donc consister à stimuler la voie secondaire du métabolisme du galactose assurant la transformation du galactose en galactonate (composé oxydable en xylulose), en augmentant soit le taux d'enzyme galactose déshydrogénase disponible pour cette réaction, soit en augmentant l'activité de l'enzyme endogène. Cela aurait pour effet de diminuer le taux de galactose et également le taux de galactose-1-phosphate intracellulaire. Cette possibilité serait envisageable sachant qu'une accumulation de xylulose n'est pas toxique pour l'organisme.

Afin de préserver les patients d'une intoxication par le galactitol, un inhibiteur de l'enzyme aldose réductase, déjà testé sur le développement de cataractes chez l'animal [42], pourrait limiter l'accumulation toxique de ce dérivé non métabolisable.

\section{Élaboration \\ d'un modèle animal expérimental de la galactosémie}

A l'heure actuelle, le seul modèle expérimental de la galactosémie utilisé est un modèle animal d'intoxication au galactose. Ce modèle expérimental consiste à nourrir un animal sain avec d'importantes doses de galactose. Le régime alimentaire enrichi à $40 \%$ en galactose déclenche l'apparition de symptômes similaires aux symptômes précoces observés chez des patients atteints de galactosémie: cataractes, troubles hépatiques et troubles du dévcloppement.

Cependant, ce modèle d'intoxication au galactose ne peut pas être considéré comme un véritable modèle animal de la galactosémic. En effet, les dent par conséquent une batterie enzymatique tout à fait fonctionnelle. Ainsi, afin de déclencher une accumulation de galactose et de ses métabolites chez ces animaux, les taux de galactose administrés sont particulièrement élevés (minimum $40 \%$ ) de manière à dépasser les capacités endogènes des enzymes impliquées dans le métabolisme du galactose. De ce fait, le galactose est non seulement phosphorylé en galactose-1-phosphate par l'enzyme GALK, mais les voies métaboliques secondaires sont également mises en jeu de manière à produire du galactitol et du galactonate à partir du galactose. Ce sont ces dérivés qui seraient responsables des symptômes précoces de l'intoxication au galactose chez les patients atteints de galactosémie avant l'instauration du régime alimentaire sans galactose.

Ainsi, à l'heure actuelle, aucun modèle animal spécifique de la galactosémie n'a été élaboré, malgré la construction d'un animal génétiquement déficient en enzyme GALT [43]. L'expérience, réalisée che\% des souris, a montré qu'une déficience totale de l'enzyme GALT est viable, et que, bien qu'accompagnée d'une accumulation de galactose et de galactose-1-phosphate dans le foie et les globules rouges, les souris ne présentent pas les symptômes d'une intoxication au galactose, qu'ils soient aigus (pas d'altération des fonctions hépatiques) ou à long terme (pas d'infertilité), observés che\% les patients atteints de galactosémie. Ceci pose le problème de la nature exacte des altérations responsables des symptômes observés chez l'homme et l'on peut supposer soit qu'il existe des mutations associées à celle de l'enzyme GALT, soit que les mécanismes de compensation font défaut dans l'espèce humaine. Ce modèle de souris génétiquement déficient en GALT caractérisé par une activité d'aldose réductase particulièrement basse pourrait être corrigé par transgénèse pour étudier la toxicité due à une association de taux élevés de galactose-1-phosphate et de galactitol.

\section{Conclusions}

Après quarante années de recherches sur la pathogénie de la galactosémie, de nouvelles données mettent en évidence l'importance des processus de glycosylation des glycoprotéines et des glycolipides et, par conséquent, confirment le rôle fondamental du métabolisme du galactose chez l'homme.

En effet, l'altération du métabolisme du galactose, par le biais de faibles taux d'UDP-galactose et d'une accumulation de galactose et de ses métabolites, entraîne une perturbation des processus de glycosylation. Celleci pourrait apparaître en particulier à une période donnée où les molécules glycosylées sont produites en quantité massive. Cela suggère très fortement le rôle du galactose et de ses métabolites dans l'apparition des complications à long terme endocriniennes et neurologiques puisque de nombreux composés liés au développement endocrinien et nerveux sont glycosylés et plus particulièrement galactosylés.

Il convient alors d'élaborer un suivi clinique des patients atteints de galactosémie, comportant des dosages systématiques de certaines molécules potenticllement toxiques comme le galactose, le galactose-1phosphate, le galactitol et le galactonate en particulier à des périodes critiques du développement. De même, il serait particulièrement utile de contrôler le niveau de glycosylation des composés impliqués dans les processus de reproduction et à certains stades de développement du système nerveux.

Enfin, l'étude approfondie des mécanismes pathogéniques de la galactosémie permet une avancée considérable quant à la compréhension des syndromes CDG, également associés à une altération de la synthèse des chaînes de hydrates de carbone de glycoprotéines et dont les mécanismes physiopathologiques restent à élucider

\section{RÉFÉRENCES}

I. MacDowell (;, (;ahl W'A. Inherited disorders of glycoprotein synthesis: cell biological insights. Proc Soc Exp Biol Med 1997; 215 : $145-57$.

2. Spaapen IJM, Vulsma T, Theunissen PMVM, Van der Meer SB, Jaeken J. (jalactosemia, a carbohydrate-deficient glycoprotein syndrome. Abstracts of the 30th Anuuat Symposium. Society for the study of inborm errors of metabolism. Lancaster: Kluwer Academic Publishers, 1992: 10. 


\section{RÉFÉRENCES}

3. Ng WG, Xu YK, Kaufman FR, Donnell GN. Deficit of uridine diphosphate galactose in galactosaemia. I Inherit Metab Dis $1989 ; 12: 257-66$.

4. Dobbie JA, Holton JB, Clamp JR. Defective galactosylation of proteins in cultured skin fibroblasts from galactosaemic patients. Ann Clin Biochem 1990; 27 : 274-5.

5. Petry KG, Greinix HT, Nudelman E, et al. Characterisation of a novel biochemical abnormality in galactosaemia : deficiency of glycolipids containing galactose or $\mathrm{N}$-acetylgalactosamine and accumulation of precursors in brain and lymphocytes. Biochem Med Metab Biol 1991 ; 46: 93-104.

6. Ornstein KS, McGuire EJ, Berry GT, Roth $\mathrm{S}$, Segal S. Abnormal galactosylation of complex carbohydrates in cultured fibroblasts from patients with galactose-1-phosphate uridyl transferase deficiency. Pediatr Res $1992 ; 31$ : 508-11.

7. Prestoz LLC, Couto AS, Shin YS, Petry KG. Altered follicle stimulating hormone isoforms in female galactosaemia patients. Eur J Pediatr 1997; 156: 116-20.

8. Von Reuss A. Zuckerausscheidung im Säuglingsalter. Wien Med Wschr 1908; 58: 799-800.

9. Göppert F. Galaktosurie nach Milchzuckergabe bei angeborenem, familiärem, chronischem Leberleiden. Klin Wochenschr $1917 ; 54: 473-5$.

10. Isselbacher KI, Anderson EP, Kuahashi $\mathrm{K}$, Kalckar HM. Congenital galactosemia, a single enzymatic block in galactose metabolism. Science 1956; 123: 635-6.

11. Reichardt JKV, Woo SLC. Molecular basis of galactosaemia: mutations and polymorphisms in the gene encoding human galactose-1-phosphate uridyl transferase. Proc Natl Acad Sci USA 1991 ; 88: 2633-7.

12. Petry KG, Reichardt JKV. The fundamental importance of human galactose metabolism: lessons from genetics and biochemistry. Trends Genet 1998; 14: 98-102.

13. Segal S, Berry GT. Disorders of galactose metabolism. In : Scriver CR, Beaudet AL, Sly WS, Valle D, eds. The metabolic and molecular bases of inherited diseases. New York: McGrawHill, 1995: 967-1000.

14. Waggoner DD, Buist NRM, Donnell GN. Long-term prognosis in galactosaemia: results of a survey of 350 cases. I Inherit Metab Dis 1990; 13: 802-18.

15. Mason HH, Turner ME. Chronic galactosemia. Am J Dis Child 1935; 50: 359-74.

16. Donnell GN, Koch R, Bergren WR. Observation on results of management of galactosemic patients. In : Hsia DYY, ed. Galactosemia. Springfield : CC Thomas, 1969: 247-70.

$m / s n^{\circ} 6-7$, vol. 16, juin-juillet 2000
17. Gitzelmann R, Hansen RG. Galactose biogenesis and disposal in galactosemics. Biochim Biophys Acta 1974; 372: 374-8.

18. Editorial. Clouds over galactosemia. Lancet 1982; 2: 1379-80.

19. Hansen TWR, Henrichsen B, Rasmussen RK, Carling A, Andreassen AB, Skjeldal O. Neuropsychological and linguistic follow-up studies of children with galactosaemia from an unscreened population. Acta Paediatr 1996; 85: 1197-201.

20. Waggoner DD, Buist NRM. Long-term complications in treated galactosaemia 175 US cases. Int Pediatr 1993; 8: 97-100.

21. Choulot IJ, Brivet M, Virlon P, et al. Évolution neurologique sévère d'une galactosémie. Défaut de myélinisation par synthèse insuffisante en UDP galactose? Arch Fr Pediatr 1991 ; 48: 267-9.

22. Schweitzer S, Shin YS, Jakobs C, Brodehl $\mathrm{J}$. Long-term outcome in 134 patients with galactosaemia. Eur I Pediatr 1993; 152: 3643.

23. Haberland C, Perou M, Brunngraber EG. The neuropathology of galactosemia : histopathological and biochemical study. $I$ Neuropathol Exp Neurol 1971 ; 30: 431-7.

24. Kaufman FR, Kogut MD, Donnell GN Goebelsmann U, March C, Koch R. Hypergonadotropic hypogonadism in female patients with galactosaemia. $N$ Engl $J$ Med $1981 ; 304: 9948$

25. Kaufman FR, Xu YK, Donnell GN. Correlation of ovarian function with galactose1-phosphate uridyl transferase levels in galactosemia. J Pediatr 1988; 112: 754-6.

26. Kaufman FR, Ng WG, Xu YK, Guidici $T$, Kaleita TA, Donnell GN. Treatment of patients (PTS) with classical galactosaemia (G) with oral uridine. Abstracts of the 27 th Annual Symposium. Society for the study of inborn errors of metabolism. Lancaster: Kluwer Academic Publishers, 1989: 8.

27. Bousfield GR, Perry WM, Ward DN Gonadotropins. Chemistry and biosynthesis. In : Knobil E, Neill JD, eds. The physiology of reproduction, 2nd ed. New York : Raven Press, 1994: 1749-92.

28. Twigg S, Wallman L, McElduff A. Clinical case seminar: the resistant ovary syndrome in a patient with galactosemia: a clue to the natural history of ovarian failure. $J$ Clin Endocrinol Metab 1996; 81 : 1329-31.

29. Tapanainen JS, Aittomäki K, Huhtaniemi IT. New insights into the role of follicle-stimulating hormone. Ann Med 1997 29: 265-6.

30. Schwarz HP, Moser H, Schild J, Zuppinger K. Hypergonadotropic hypogonadism in two sisters with galactosaemia. Arch Dis Child 1984; 59: 781-3.

31. Shin YS, Rachwalik M, Podskarbi T Pathogenic mechanisms of long-term complications in classical galactosemia. In: Shin
YS, Demirkol M, eds. Diagnosis and treatment of inborm errors of metabolism : contributions to an equal opportunity for children in Asia and Europe. Istanbul: TSPKU, 1996: 108-14.

32. Gitzelmann R, Hansen RG, Steinmann B. Biogenesis of galactose, a possible mechanism of self-intoxication in galactosaemia. In: Hommes Frits A, Van den Berg CJ, eds. Normal and pathological development of energy metabolism. New York-London: Academic Press, 1975: 25-37.

33. Berry GT, Nissim I, Lin Z, Mazur AT, Gibson JB, Segal S. Endogenous synthesis of galactose in normal men and patients with hereditary galactosaemia. Lancet $1995 ; 346$ : $1073-4$

34. Van Heyningen R. Galactose cataract: a review. Exp Eye Res 1971 ; 11 : 415-28.

35. Gross KC, Acosta PB. Fruits and vegetables are a source of galactose: implications of planning the diets of patients with galactosemia. J Inherit Metab Dis 1991 ; 14: 253-8.

36. Stewart M, Kurien M, Sherman W, Cotlier $\mathrm{E}$. Inositol changes in nerve and lens of galactose-fed rats. I Neurochem 1968; 15 : 941-6.

37. Van Pelt J, van der Meer SB, Bakker JA Spaapen LJ. Carbohydrate deficient transferrin (CDT) and galactosaemia. I Inherit Metab Dis Suppl 1996; 19: 65.

38. Stibler H, von Döbeln U, Kristiansson B, Guthenberg C. Carbohydrate-deficient transferrin in galactosaemia. Acta Paediatr 1997; 86: 1377-8.

39. Manis FR, Cohn LB, McBride-Chang C, Wolff JA, Kaufman FR. A longitudinal study of cognitive functioning in patients with classical galactosaemia, including a cohor treated with oral uridine. I Inherit Metab Dis $1997 ; 20: 549-55$.

40. Rogers SR, Segal S. Modulation of rat tissue galactose-1-phosphate uridyl transferase by uridine triphosphate. Pediatr Res $1991 ; 30: 222-6$.

41. Prestoz LLC, Daude N, Cournu I, Shin YS, Petry KG. Differential UDP-galactose-4'epimerase (GALE) enzymatic activity and mRNA expression in the rat mammary gland during lactation. FEBS Lett 1998; 431 . 391-4.

42. Robinson WG Jr, Laver NM, Jacot JL, et al. Diabetic-like retinopathy with the aldose reductase inhibitor WAY-121,590. Invest Ophthalmol Vis Sci 1996; 37: 1149-56.

43. Leslie ND, Yager KL, McNamara PD, Segal S. A mouse model of galactose-1-phosphate uridyl transferase deficiency. Biochem Mol Med 1996; 59: 7-12.

\section{TIRÉS À PART}

K.(;. Petr? 


\section{Summary}

A century of galactosaemia research : the fundamental role of carbohydrates in glycoconjugates

This work presents an overview of the scientific and clinical research carried out on galactosaemia in the last decades and the recent data reported in this field. Galactosaemia is an inborn error of galactose metabolism. This disease is caused by mutations localised on the gene encoding the enzyme galactose-1phosphate uridyl transferase (GALT) playing a key role in galactose metabolism. Galactosaemic patients exhibit long term complications such as symptoms of neurodegenerative disease as well as severe endocrinologic troubles. Pathogenic mechanisms of these clinical manifestations are still poorly understood but some recent data strongly support that an alteration in the glycosylation process of glycoproteins and glycolipids is involved in the development of these troubles. More precisely, an altered glycosylation process could be caused not only by a metabolic deficit due to the enzymatic deficiency but also by the accumulation of metabolites upstream to the deficient enzymatic reaction. The research work carried out on galactosaemia emphasises the fundamental role of carbohydrate chains of glycoproteins and glycolipids and is essential for the understanding of carbohydrate deficient glycoprotein (CDG) syndromes, that are still poorly understood.
I EURESCO CONFERENCES OF THE EUROPEAN SCIENCE FOUNDATION

- The Science of Aphasia

EuroConference : from therapy to theory

I. PAPATHANASIOU (Londres)

8-13 septembre 2000 - Aghia Pelaghia, Crète, Grèce

- Brain development and cognition in human infants : EuroConference on normal and abnormal cortical functional specializations

C. BUTTERWORTH (Brighton) - F. SIMION (Padova)

15-20 septembre 2000 - La-Londe-les-Maures

- B Cells in Health and Disease : B-cell Tumors

K. NILSSON (Uppsala)

3-8 octobre 2000 - Lenggries (near Munich) Allemagne

- Communication within the Immune System : Basic rules and their breakdown : Euroconference on Molecular Determinants of Tolerance and autoimmunity

A. COOKE (Cambridge)

6-11 octobre 2000 - Obernai

\section{Renseignements EURESCO Conferences \\ European Science Foundation,}

1, quai Lezay-Marnézia, 67080 Strasbourg Cedex - Fax : 0388366987

E-mail : euresco@esf.org

http://www.esf.org/euresco
BOURSES ET SUBVENTIONS DE RECHERCHE 2000-2001

PARMI LES DOMAINES SUIVANTS :

GÉNÉTIQUE MÉDICALE (Corrélations génotype-phénotype, Épidémiologie Génétique...)

RECHERCHE CLINIQUE ET THÉRAPEUTIQUE

(Consultations pluridisciplinaires et Réseaux de Soins...)

SCIENCES SOCIALES (Droit, Économie, Éthique, Psychologie...)

CONCERNANT LES FORMES GÉNÉTIQUES DES MALADIES ORPHELINES DE L'AFRG :

Aicardi, Amyloses, Angelman, AxenfeldRieger, Behçet, Chorée de Huntington, CADASIL, CDG, Délétions chromosomiques, Dysplasies-Ectodermiques, Dysplasies osseuses avec retard, Ehlers-Danlos, Fibrohyalinose juvénile, Fibromyalgie, Gilles de la Tourette, Hémiplégie alternante du Nourrisson, Hémoglobinurie Paroxystique Nocturne, Hidrosadénite suppurée, Histiocytose, Homme de Pierre, Insuffisance des Surrénales, Leucodystrophies sans cause, Lowe, Maladies du Métabolisme des Protéines (Homocystinuries), Marfan, McCune-Albright, Moebius, Naevus, Neurofibromatoses, Noonan, Ollier-Maffucci, Ostéogenèse Imparfaite, Paralysie Supra-Nucléaire Progressive, PseudoObstruction Intestinale Chronique, QT Long, Rétinoblastome, Sarcoidose, Trisomies, Von-Hippel Lindau, Wilson.

\section{DEA ET FIN DE THESE}

Trois bourses de recherche $(80000 \mathrm{~F}$ chacune) seront attribuées en collaboration avec les laboratoires BIOGALENIQUE-RPG.

POST-DOCTORAT ET SUBVENTIONS

Trois bourses post-doctorales ou subventions de recherche $(80000 \mathrm{~F}$ chacune) seront attribuées pour un an avec le concours de CONTINENT Groupe Promodès.

DOSSIERS A RETIRER AUPRÈS DE L'AFRG

Date limite de dépôt : 15 septembre 2000

Renseignements : Monsieur Alain BOUVET

AFRG, 5, rue Casimir-Delavigne 75006 Paris Tél. : 0143259800 - Fax : 0143543256 $\mathrm{N}^{\circ}$ INDIGO : 0820800008

e-mail: mmo@afrg.org http://www.afrg.org 